\title{
Corrigendum: Association Between Sleep Disorders and Cognitive Impairment in Middle Age and Older Adult Hemodialysis Patients: A Cross-Sectional Study
}

\section{OPEN ACCESS \\ Approved by: Frontiers Editorial Office, \\ Frontiers Media SA, Switzerland \\ *Correspondence: \\ Yang Luo \\ luoy@bisith.cn \\ †These authors have contributed equally to this work and share first authorship}

Specialty section:

This article was submitted to Neurocognitive Aging and Behavior, a section of the journal

Frontiers in Aging Neuroscience

Received: 09 December 2021 Accepted: 10 December 2021

Published: 05 January 2022

Citation:

Tian R, Bai Y, Guo Y, Ye P and Luo Y (2022) Corrigendum: Association

Between Sleep Disorders and Cognitive Impairment in Middle Age and Older Adult Hemodialysis Patients: A Cross-Sectional Study Front. Aging Neurosci. 13:831897. doi: 10.3389/fnagi.2021.831897

\section{Ru Tian ${ }^{1 t}$, Yun Bai ${ }^{2 t}$, Yidan Guo ${ }^{1}$, Pengpeng $\mathrm{Ye}^{3}$ and Yang Luo ${ }^{1 *}$}

\begin{abstract}
${ }^{1}$ Division of Nephrology, Beijing Shijitan Hospital, Capital Medical University, Bejing, China, ${ }^{2}$ Department of Obstetrics and Gynecology, Beijing Jishuitan Hospital, Beijing, China, ${ }^{3}$ Division of Injury Prevention and Mental Health, National Center for Chronic and Non-communicable Disease Control and Prevention, Chinese Center for Disease Control and Prevention, Beijing, China
\end{abstract}

Keywords: sleep, sleep disorders, cognitive function, cognitive impairment, hemodialysis

\section{A Corrigendum on}

Association Between Sleep Disorders and Cognitive Impairment in Middle Age and Older Adult Hemodialysis Patients: A Cross-Sectional Study

by Tian, R., Bai, Y., Guo, Y., Ye, P., and Luo, Y. (2021). Front. Aging Neurosci. 13:757453. doi: 10.3389/fnagi.2021.757453

In the published article, there was an error regarding the co-author affiliation. Instead of "Ru Tian", Yun Bai ${ }^{2 "}$, it should be "Ru Tian ${ }^{1+}$, Yun $\mathrm{Bai}^{2+"}$ with the note "+ These authors have contributed equally to this work and share the first authorship".

The authors apologize for this error and state that this does not change the scientific conclusions of the article in any way. The original article has been updated.

Publisher's Note: All claims expressed in this article are solely those of the authors and do not necessarily represent those of their affiliated organizations, or those of the publisher, the editors and the reviewers. Any product that may be evaluated in this article, or claim that may be made by its manufacturer, is not guaranteed or endorsed by the publisher.

Copyright ( 2022 Tian, Bai, Guo, Ye and Luo. This is an open-access article distributed under the terms of the Creative Commons Attribution License (CC BY). The use, distribution or reproduction in other forums is permitted, provided the original author(s) and the copyright owner(s) are credited and that the original publication in this journal is cited, in accordance with accepted academic practice. No use, distribution or reproduction is permitted which does not comply with these terms. 